these clinical findings, this patient was considered to have idiopathic constrictive pericarditis in New York Heart Association (NYHA) class IV. Pericardectomy was indicated. We planned to monitor the $\mathrm{p}-\mathrm{v}$ loops of the left ventricle during operation to evaluate the effects of the procedure.

In December 1994, an operation was performed through a median sternotomy. A catheter-tip micromanometer and a conductance catheter were introduced into the left ventricle through the ascending aorta. The preoperative p-v loops showed an unusual shape (Fig. 1, $b$ ) compared with normal p-v loops (Fig. 1, a). After the removal of the pericardium between the right phrenic nerve and the left phrenic nerve, a small degree of improvement was noted on the p-v loops (Fig. 1,c), but the shape remained basically the same as before operation. We added resection of the thickened pericardium attached to the lateral and the inferior walls of the left ventricle under cardiopulmonary bypass with cannulation of the right atrium and the femoral artery. The p-v loops after weaning from bypass were almost normal (Fig. 1, d). We considered the operations successful.

The postoperative course was generally good. In the early phase after operation, the patient needed relatively high doses of diuretics and plasma products. Thereafter, ascitic fluid gradually decreased and subjective symptoms showed obvious improvement. Cardiac catheterization after 42 postoperative days showed obvious improvement: right atrium, $12 \mathrm{~mm} \mathrm{Hg}$; right ventricle, $33 / \sim 12 \mathrm{~mm} \mathrm{Hg}$; and left ventricle, $105 / \sim 15 \mathrm{~mm} \mathrm{Hg}$. Blood chemistry test revealed a manifest decrease of alkaline phosphatase ( 660 U/L, compared with $1086 \mathrm{U} / \mathrm{L}$ before operation). The patient's body weight and the girth of his abdomen reduced and were almost stable $(56 \mathrm{~kg}$ and $79 \mathrm{~cm}$, respectively) with furosemide (Lasix) at a dosage of $80 \mathrm{mg}$ orally. The patient was discharged after 45 postoperative days.

Clinical results of pericardectomy (mainly between the right phrenic nerve and the left phrenic nerve) for constrictive pericarditis are generally stable and good for patients in NYHA classes I, II, and III, whereas results for patients in NYHA class IV are usually poor. ${ }^{3,4}$ In addition, there is persistent controversy regarding the optimal extent of pericardial resection for each case, and evaluating the effects of resection is difficult during operation. We therefore planned to monitor the $p-v$ loops of the left ventricle for this patient in NYHA class IV to evaluate the effects of the procedure and to determine the optimal extent of pericardial resection. Intraoperative normalization of the p-v loops led us to expect a good postoperative course. In line with our expectations, NYHA class (postoperative class II), ascitic level, and clinical data had improvement obvious and were stable after operation.

This case showed that intraoperative monitoring of the p-v loops of the left ventricle, easily performed by the conductance catheter method, expresses well the cardiodynamics of the individual heart and is an excellent and useful technique for evaluating the effects of pericardectomy for constrictive pericarditis.

\section{REFERENCES}

1. Baan J, Aouw TT, Kerkhof PL, et al. Continuous stroke volume and cardiac output from interventricular dimensions obtained with impedance catheter. Cardiovasc Res 1981;15:328-34.

2. Baan J, Verde ET, Bruin HG, et al. Continuous measurement of left ventricular volume in animals and humans by conductance catheter. Circulation 1984;70:812-23.

3. Devaleria PA, Baumgartner WA, Casale AS, et al. Current indication, risks, and outcome after pericardectomy. Ann Thorac Surg 1991;52:219-24.

4. Tirilomis T, Unverdorben S, Emide J. Pericardectomy for chronic constrictive pericarditis. Eur J Cardiothorac Surg 1994;8:487-92.

\title{
TRANSAORTIC VIDEO-ASSISTED EXCISION OF A LEFT VENTRICULAR PAPILLARY FIBROELASTOMA
}

Keith B. Allen, MD, ${ }^{a}$ Marshall Goldin, $\mathrm{MD},{ }^{\mathrm{b}}$ and Ramon Mitra, $\mathrm{MD}, \mathrm{PhD},{ }^{\mathrm{c}}$ Indianapolis, Ind., and Chicago, Ill.

Papillary fibroelastomas are rare intracardiac tumors that can arise from any endocardial surface. Before the advent of echocardiography, diagnosis was primarily an

From the Department of Cardiovascular-Thoracic Surgery, St. Vincent Medical Center, Indianapolis, Ind., and the Departments of Cardiovascular-Thoracic Surgery, ${ }^{b}$ and Cardiology, ${ }^{c}$ Rush-Presbyterian-St. Luke's Medical Center, Chicago, Ill.

Received for publication August 22, 1995; accepted for publication Sept. 13, 1995.

J Thorac Cardiovasc Surg 1996;112:199-201

Copyright (c) 1996 by Mosby-Year Book, Inc.

$0022-5223 / 96 \$ 5.00+0 \quad \mathbf{1 2 / 5 4 / 6 9 2 6 2}$ incidental finding at autopsy or operation. Despite the diminutive size of papillary fibroelastomas, surgical excision is generally indicated to prevent life-threatening coronary, cerebral, and systemic emboli. This article reports the first video-assisted removal of an intracardiac tumor.

A 57-year-old white man was admitted for evaluation of recurrent atrial fibrillation. Previous operations included myomectomy for idiopathic hypertrophic subaortic stenosis 10 years before this admission. History, results of physical examination, and laboratory profile were unremarkable. Despite appropriate medical therapy, the patient remained in atrial fibrillation.

Before elective cardioversion, a two-dimensional echo- 


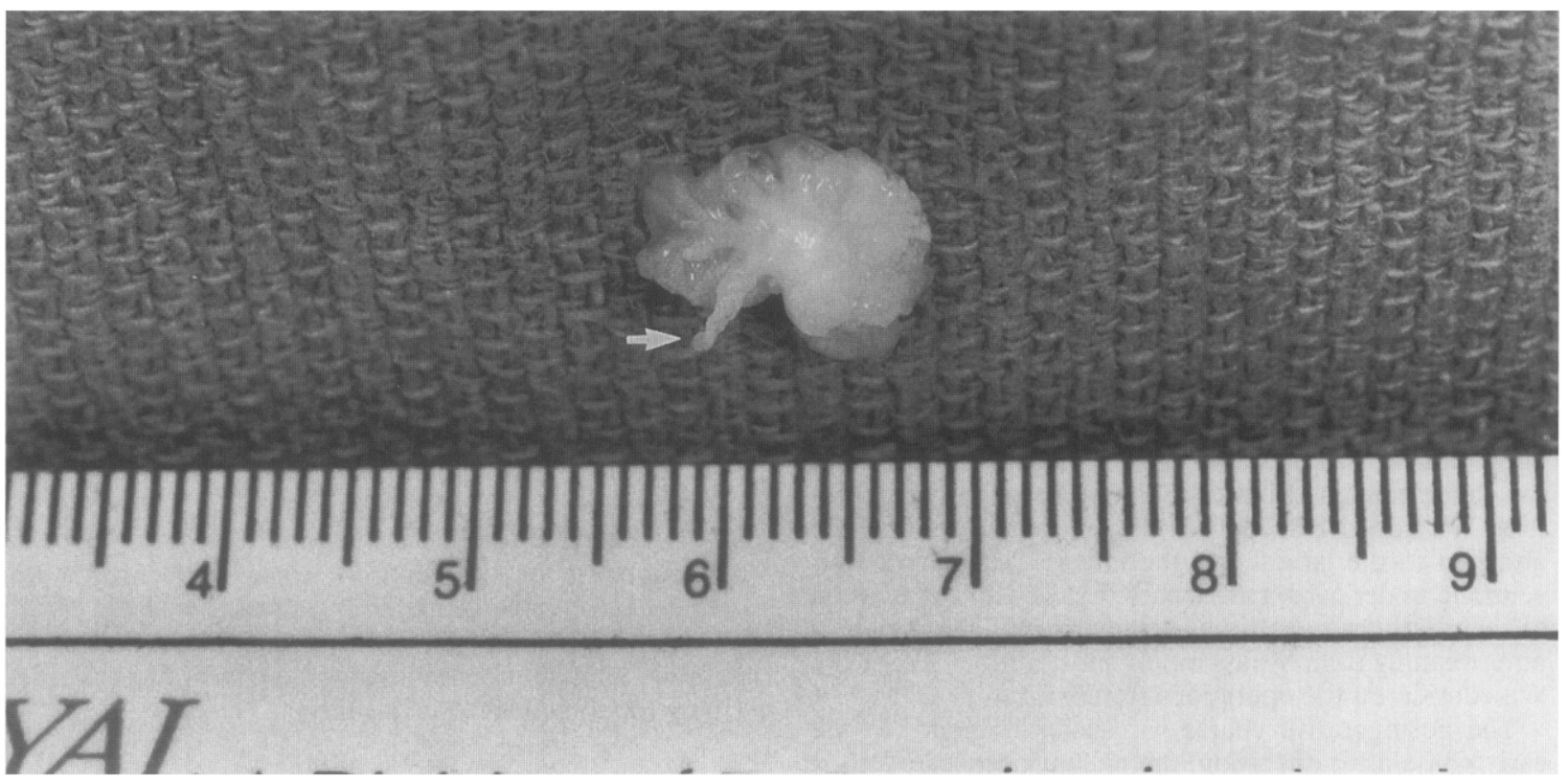

Fig. 1. Gross appearance of the excised papillary fibroelastoma with its attached stalk (arrow).

cardiographic scan unexpectedly demonstrated a stalked $1.5 \times 1.5 \mathrm{~cm}$ mass arising from the posterior medial papillary muscle. Echocardiographic diagnosis was consistent with a small myxoma or papillary fibroelastoma. Cardiac catheterization demonstrated normal coronary anatomy and no evidence of left ventricular outflow tract obstruction. Surgical excision was recommended in consideration of the known potential for coronary, cerebral, and systemic embolization.

Sternotomy was uneventful; bicaval and ascending aortic cannulation were accomplished and total cardiopulmonary bypass was initiated with systemic cooling to $30^{\circ} \mathrm{C}$. The small left atrial size made the standard left atrial approach less desirable; a transaortic approach was chosen to avoid a ventricular incision, with its potential complications.

With the patient in cardioplegic arrest, a hockey-stick incision was created in the ascending aorta; however, transvalvular inspection of the left ventricular cavity did not demonstrate the mass. With standard videothorascopic equipment, a viewing scope that allowed simultaneous visualization and biopsy was passed through the aortic valve and into the left ventricle. A pedicled mass with delicate papillary fronds resembling a sea anemone was seen arising from the endocardial surface at the base of the posterior medial papillary muscle. The stalk of the mass was easily grasped with the biopsy portion of the viewing scope, allowing excision of the entire tumor (Fig. 1). Intraoperative transesophageal echocardiography demonstrated no residual mass.

The specimen measured $1.5 \times 1.5 \mathrm{~cm}$, with the papillary fronds averaging 0.5 to $1 \mathrm{~cm}$ in length. Histologically, the papillary fronds consisted of a central core of dense connective tissue surrounded by a layer of loose connec- tive tissue covered by hyperplastic endothelial cells. Results of gross and histologic examinations were consistent with the final diagnosis of papillary fibroelastoma.

The postoperative course was uncomplicated, with the exception of recurrent atrial fibrillation necessitating cardioversion. Patient follow-up at 20 months demonstrated no evidence of tumor recurrence.

Papillary fibroelastomas are rare, characteristically small, nonneoplastic tumors representing $1 \%$ to $4 \%$ of all intracardiac masses. ${ }^{1}$ Although infrequently seen in patients younger than 50 years, papillary fibroelastomas are the most common type of tumor arising from cardiac valves and have been reported to arise from any endocardial surface. ${ }^{2}$

Before the advent of echocardiography, incidental diagnosis was occasionally made at the time of heart operations; however, such tumors were usually pathologic curiosities discovered at autopsy. The techniques of twodimensional echocardiography and, more recently, transesophageal echocardiography provide both a definitive preoperative diagnosis and a means of accurate postoperative follow-up. ${ }^{3}$ Cardiac catheterization is still recommended to exclude associated coronary artery disease; however, left ventriculography should be avoided to prevent iatrogenic tumor embolization.

Grossly, these tumors resemble sea anemones, with numerous villuslike projections extending from a central stalk. Histologically, the papillary fronds typically have three distinct architectural zones: (1) a central core consisting of dense connective tissue, (2) a peripheral loose myxomatous zone with a rich mucopolysaccharide matrix, and (3) an overlying layer of hyperplastic endothelium. This last layer of endothelium is contiguous with the endothelial cells lining the endocardium or cardiac valves. 
Despite their small size, papillary fibroelastomas have been reported to cause angina and sudden death from either coronary emboli or direct ostial occlusion by prolapsing tumor. ${ }^{4}$ In addition, cerebral embolization and infarction have been reported to be caused by tumor particles or, more likely, fragmentation of thrombus, which has a propensity toward accumulation on the extensive surface area provided by the tumor fronds. ${ }^{3,5}$ Although papillary fibroelastomas are considered "benign," their clinical course is deceptive. In light of their unpredictable nature, papillary fibroelastomas should be resected even in the patient who has no symptoms.

\section{REFERENCES}

1. McAllister HA, Fenogolio JJ. Tumors of the cardiovascular system. In: Atlas of tumor pathology, 2nd series, fascicle 15.
Washington DC: Armed Forces Institute of Pathology, 1978: 20-5.

2. Edwards FH, Hale D, Cohen A, Thompson L, Pezzella AT, Virmani R. Primary cardiac valve tumors. Ann Thorac Surg 1991;52:1127-31.

3. Ong LS, Nanda NC, Barold SS. Two-dimensional echocardiographic detection and diagnostic features of left ventricular papillary fibroelastoma. Am Heart J 1982;103:917-8.

4. Boone S, Higginson LA, Walley VM. Endothelial papillary fibroelastomas arising in and around the aortic sinus, filling the ostium of the right coronary artery. Arch Pathol Lab Med 992;116:135-7.

5. McFadden PM, Lacy JR. Intracardiac papillary fibroelastoma: an occult cause of embolic neurologic deficit. Ann Thorac Surg 1987;43:667-9. 